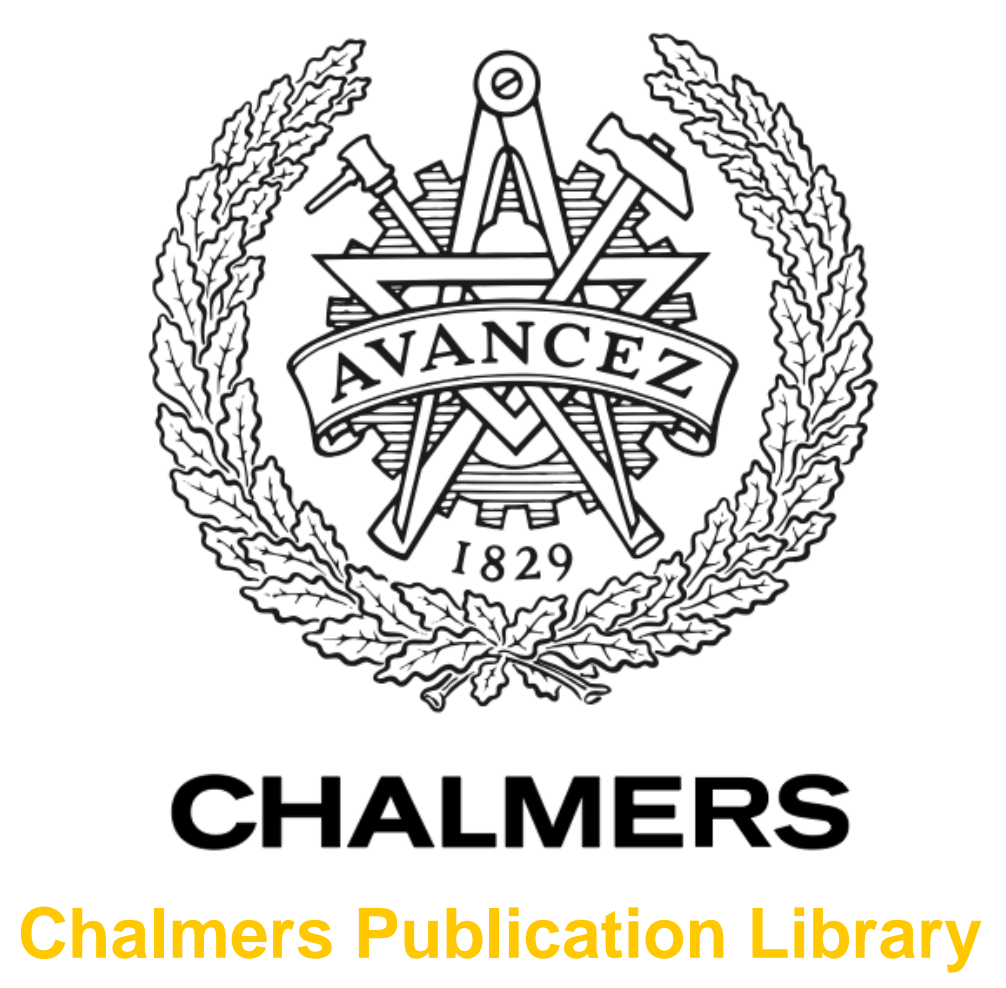

\title{
Channel gain prediction for multi-agent networks in the presence of location uncertainty
}

This document has been downloaded from Chalmers Publication Library $(\mathrm{CPL})$. It is the author's version of a work that was accepted for publication in:

41st IEEE International Conference on Acoustics, Speech and Signal Processing, ICASSP 2016, Shanghai, China, 20-25 March 2016 (ISSN: 1520-6149)

Citation for the published paper:

Fröhle, M. ; Muppirisetty, L. ; Wymeersch, H. (2016) "Channel gain prediction for multiagent networks in the presence of location uncertainty". 41st IEEE International Conference on Acoustics, Speech and Signal Processing, ICASSP 2016, Shanghai, China, 20-25 March 2016 pp. 3911-3915.

http://dx.doi.org/10.1109/ICASSP.2016.7472410

Downloaded from: http://publications.lib.chalmers.se/publication/233822

Notice: Changes introduced as a result of publishing processes such as copy-editing and formatting may not be reflected in this document. For a definitive version of this work, please refer to the published source. Please note that access to the published version might require a subscription. 


\title{
CHANNEL GAIN PREDICTION FOR MULTI-AGENT NETWORKS IN THE PRESENCE OF LOCATION UNCERTAINTY
}

\author{
Markus Fröhle, L. Srikar Muppirisetty, Henk Wymeersch \\ Department of Signals and Systems, Chalmers University of Technology, Gothenburg, Sweden \\ E-mail:\{frohle, srikar.muppirisetty, henkw\}@chalmers.se
}

\begin{abstract}
Coordination among mobile agents relies on communication over a wireless channel and can thus be improved by channel prediction. We present a Gaussian process framework to learn channel parameters and predict the channel between arbitrary transmitter and receiver locations. We explicitly incorporate location uncertainty in both learning and prediction phases. Simulation results show that if location uncertainty is not modeled appropriately, it has a degenerative effect on the prediction quality.
\end{abstract}

Index Terms - Multi-agent network, channel prediction, Gaussian processes, location uncertainty

\section{INTRODUCTION}

When performing a task, mobile agents need to be aware of their own location and communicate with each other over the wireless channel. Tasks can include formation control and goal-seeking [1], which requires explicit communication and connectivity maintenance. Prediction of channel gains can improve connectivity and system performance [2]. The channel gain in a wireless channel comprises three main components: deterministic path-loss, random shadowing, and random small-scale fading [3]. Small-scale fading decorrelates over distances on the order of a few centimeters (and will be considered averaged out in this study), whereas shadowing decorrelates over 50-100 m outdoors [3] and 1-5 $\mathrm{m}$ indoors $[4,5]$, with well-established shadowing correlation models $[6,7]$. In this paper, we adapt the Gaussian process (GP) model of [8] to incorporate uncertain location information at both communicating endpoints. Our framework is able to deal with channel measurements recorded at uncertain locations and to incorporate this location uncertainty into the predictive distribution of the wireless channel at a given (possibly uncertain) test location.

This work is supported, in part, by the European Research Council under Grant No. 258418 (COOPNET), and by EU FP7 Marie Curie Initial Training Network MULTI-POS (Multi-technology Positioning Professionals) under Grant No. 316528.

\section{RELATION TO PRIOR WORK}

Modeling of multi-agent wireless channels was considered in [9], where shadowing was modeled using a spatial loss field, integrated along a line between transmitter and receiver. Prediction of wireless channels between agents was studied in [10,11], where [10] proposed a GP framework, while [11] investigated the impact of the underlying channel parameters on the spatial channel prediction variance. A common assumption in [9-11] was the presence of perfect location information. This assumption was partially removed in [12], which extends [11] to quantify the effect of localization errors on spatial channel prediction. For the case of a fixed transmitter location, [8] explicitly accounted for location uncertainty at the receiver, but not at the transmitter, using a GP framework.

\section{MODEL AND PROBLEM STATEMENT}

\subsection{Channel Model}

We consider a TX and RX with locations $\mathbf{q}_{\mathrm{TX}} \in \mathbb{R}^{D}$ and $\mathbf{q}_{\mathrm{RX}} \in \mathbb{R}^{D}$, respectively, where $D$ is the dimensionality of the space. The transmitted power is fixed to $P_{\mathrm{TX}}$. Assuming measurements average out small-scale fading, either in time (measurements taken over a time window) or frequency (measurements represent average power over a large frequency band), the received signal power in $\mathrm{dBm}$ can be expressed as [3]

$$
\begin{aligned}
& P_{\mathrm{RX}}\left(\mathbf{q}_{\mathrm{TX}}, \mathbf{q}_{\mathrm{RX}}\right)=P_{\mathrm{TX}}+L_{0} \\
& -10 \eta \log _{10} \frac{\left\|\mathbf{q}_{\mathrm{TX}}-\mathbf{q}_{\mathrm{RX}}\right\|}{d_{0}}+\Psi\left(\mathbf{q}_{\mathrm{TX}}, \mathbf{q}_{\mathrm{RX}}\right),
\end{aligned}
$$

where constant $L_{0}$ captures antenna and other propagation gains, $d_{0}$ is a reference distance (here set to $1 \mathrm{~m}$ ), $\eta$ denotes the path-loss exponent, and shadowing (in the $\mathrm{dB}$ domain) is modeled through a zero-mean normal distribution with spatial correlation $[6,13]$ and channel reciprocity (i.e., $\Psi\left(\mathbf{q}_{\mathrm{TX}}, \mathbf{q}_{\mathrm{RX}}\right)$ $\left.=\Psi\left(\mathbf{q}_{\mathrm{RX}}, \mathbf{q}_{\mathrm{TX}}\right)\right)$. For notational convenience, we will denote the positions of a TX-RX pair by $\mathbf{x}=\left[\mathbf{q}_{\mathrm{TX}}^{\mathrm{T}}, \mathbf{q}_{\mathrm{RX}}^{\mathrm{T}}\right]^{\mathrm{T}} \in \mathbb{R}^{2 D}$, allowing us to write $P_{\mathrm{RX}}(\mathbf{x})$. 


\subsection{Problem Statement}

Based on the transmission from TX, RX obtains a noisy measurement $y=P_{\mathrm{RX}}(\mathbf{x})+n$, where $n \sim \mathcal{N}_{n}\left(0, \sigma_{n}^{2}\right)$. The locations of both, TX and RX, are known only statistically, through the probability density functions (pdfs) $p\left(\mathbf{q}_{\mathrm{TX}}\right)$ and $p\left(\mathbf{q}_{\mathrm{RX}}\right)$, which are assumed to be described by a finite number of parameters $\mathbf{u}$ (e.g., means and covariances of both agents' locations). We assume that $N$ measurements $\mathbf{y}=\left[y_{1}, y_{2}, \ldots, y_{N}\right]^{\mathrm{T}}$ have been collected at different TX and RX positions. We denote the corresponding location parameters $\mathbf{U}=\left[\mathbf{u}_{1}^{\mathrm{T}}, \mathbf{u}_{2}^{\mathrm{T}}, \ldots, \mathbf{u}_{N}^{\mathrm{T}}\right]^{\mathrm{T}}$, wherein $\mathbf{u}_{i}$ describes the distribution of $\mathbf{x}_{i}$, i.e., the TX and RX pair of the $i$-th measurement $y_{i}$. We further consider a new location pair distribution $\mathbf{u}_{*}$ (e.g., a possible future location for a TX and RX pair). We can now formulate the following problems:

(i) learn the parameters of the channel (say $\theta$, to be specified in the next section) from the database $\{\mathbf{U}, \mathbf{y}\}$,

(ii) determine the predictive pdf $p\left(P_{\mathrm{RX}}\left(\mathbf{u}_{*}\right) \mid \mathbf{y}, \mathbf{U}, \mathbf{u}_{*} ; \theta\right)$.

\section{CHANNEL PREDICTION}

In the following, we adapt the GP framework of [8], called uncertain GP (uGP), for learning and prediction of the wireless channel considering TX and RX location uncertainty. The received power at location pair $\mathbf{x}$ (described by its distribution parameters $\mathbf{u}$ ) is modeled as

$$
P_{\mathrm{RX}}(\mathbf{u}) \sim \mathcal{G P}\left(\mu(\mathbf{u}), k\left(\mathbf{u}, \mathbf{u}^{\prime}\right)\right) .
$$

For the mean function we select

$$
\begin{aligned}
\mu(\mathbf{u}) & =\mathbb{E}_{\mathbf{x}, \Psi}\left\{P_{\mathrm{RX}}(\mathbf{x})\right\} \\
& =P_{\mathrm{TX}}+L_{0}-10 \eta \mathbb{E}_{\mathbf{x}}\left\{\log _{10} \frac{\left\|\mathbf{q}_{\mathrm{TX}}-\mathbf{q}_{\mathrm{RX}}\right\|}{d_{0}}\right\},
\end{aligned}
$$

while a suitable choice for the covariance function is

$$
k\left(\mathbf{u}, \mathbf{u}^{\prime}\right)=\iint c\left(\mathbf{x}, \mathbf{x}^{\prime}\right) p(\mathbf{x}) p\left(\mathbf{x}^{\prime}\right) \mathrm{d} \mathbf{x} \mathrm{d} \mathbf{x}^{\prime},
$$

in which $c\left(\mathbf{x}, \mathbf{x}^{\prime}\right)$ is a covariance function under perfect location information. Here, $\mathrm{x}$ denotes the TX and RX locations of one link, and $\mathrm{x}^{\prime}$ the TX and RX locations of another link. Note that the choices (3)-(5) imply that for the case of no location uncertainty, uGP reverts back to a classical GP (cGP) approach, with classical mean and covariance functions [10].

\subsection{Selection of Mean and Covariance Functions}

We will assume that $\mu(\mathbf{u})$ can be expressed in closed form. ${ }^{1}$ For the covariance function, we first define $c\left(\mathbf{x}, \mathbf{x}^{\prime}\right)$. A rea-

\footnotetext{
${ }^{1}$ For instance, with $d=\left\|\mathbf{q}_{\mathrm{TX}}-\mathbf{q}_{\mathrm{RX}}\right\|$, a polynomial expansion of $\mu(d)=P_{\mathrm{TX}}+L_{0}-10 \eta \log _{10}(d)$ combined with a Gaussian approximation of $p(d)$ can allow a closed-form expression of $\mu(\mathbf{u})$ [8].
}

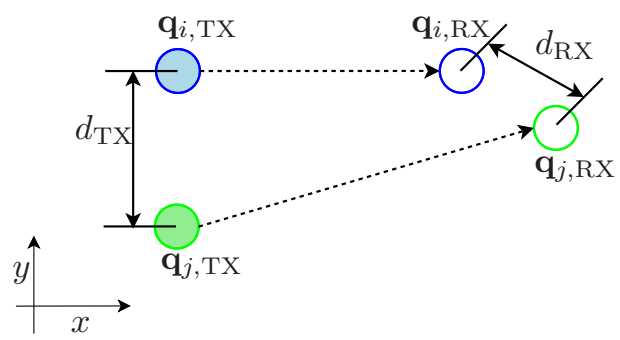

Fig. 1. Illustration of TX and RX displacement between two TX-RX pairs $i$ and $j$ in $\mathbb{R}^{2}$. The TX displacement is $d_{\mathrm{TX}}=$ $\left\|\mathbf{q}_{i, \mathrm{TX}}-\mathbf{q}_{j, \mathrm{TX}}\right\|$ and the RX displacement is $d_{\mathrm{RX}}=\| \mathbf{q}_{i, \mathrm{RX}}-$ $\mathbf{q}_{j, \mathrm{RX}} \|$.

sonable form of covariance function is [7]

$$
\begin{aligned}
c\left(\mathbf{x}, \mathbf{x}^{\prime}\right) & =\sigma_{\Psi}^{2} \exp \left(-\frac{\left\|\mathbf{q}_{\mathrm{TX}}-\mathbf{q}_{\mathrm{TX}}^{\prime}\right\|^{p}}{d_{c}^{p}}\right) \\
& \times \exp \left(-\frac{\left\|\mathbf{q}_{\mathrm{RX}}-\mathbf{q}_{\mathrm{RX}}^{\prime}\right\|^{p}}{d_{c}^{p}}\right)+\delta_{\left\{\mathbf{x}=\mathbf{x}^{\prime}\right\}} \sigma_{\text {proc }}^{2}
\end{aligned}
$$

where $\delta_{\mathrm{P}}=1$ when $\mathrm{P}=$ true and 0 otherwise, $p \geq 1, \sigma_{\Psi}^{2}$ captures the variance of the shadowing process, $\sigma_{\text {proc }}^{2}$ models any white noise not due to measurement noise (e.g., caused by kernel mismatch), and $d_{c}$ is the decorrelation distance. The first exponential in (6) corresponds to the TX displacement between $\mathbf{x}$ and $\mathbf{x}^{\prime}$ and the second exponential to the RX displacement. $^{2}$ This is illustrated in Fig. 1. With $p=1$ we have the model of [7], and if either $\mathbf{q}_{\mathrm{TX}}=\mathbf{q}_{\mathrm{TX}}^{\prime}$ or $\mathbf{q}_{\mathrm{RX}}=\mathbf{q}_{\mathrm{RX}}^{\prime}$, we get the Gudmundson model [6]. With $p=2$, it can be verified that when $\mathbf{x}$ and $\mathrm{x}^{\prime}$ are Gaussian random variables (so that $\mathbf{u}$ and $\mathbf{u}^{\prime}$ describe the means and covariances of the two TX and $\mathrm{RX}$ locations), $k\left(\mathbf{u}, \mathbf{u}^{\prime}\right)$ can be computed in closed form (see also [15, App. C] and [16, Ch. 3.4]):

$$
\begin{aligned}
& k\left(\mathbf{u}, \mathbf{u}^{\prime}\right)=\delta_{\left\{\mathbf{u}=\mathbf{u}^{\prime}\right\}} \sigma_{\text {proc }}^{2}+\sigma_{\Psi}^{2}\left|\Gamma_{\mathrm{TX}}\right|^{-1 / 2}\left|\Gamma_{\mathrm{RX}}\right|^{-1 / 2} \\
& \exp \left(-\frac{1}{d_{c}^{2}} \Delta_{\mathrm{TX}}^{\mathrm{T}} \Gamma_{\mathrm{TX}}^{-1} \Delta_{\mathrm{TX}}\right) \exp \left(-\frac{1}{d_{c}^{2}} \Delta_{\mathrm{RX}}^{\mathrm{T}} \Gamma_{\mathrm{RX}}^{-1} \Delta_{\mathrm{RX}}\right),
\end{aligned}
$$

where $\Gamma_{\mathrm{RX}}=\left(\mathbf{I}+d_{c}^{-2}\left(\Sigma_{\mathrm{RX}}+\Sigma_{\mathrm{RX}}^{\prime}\right)\left(1-\delta_{\left\{\mathbf{u}=\mathbf{u}^{\prime}\right\}}\right)\right), \Gamma_{\mathrm{TX}}=$ $\left(\mathbf{I}+d_{c}^{-2}\left(\Sigma_{\mathrm{TX}}+\Sigma_{\mathrm{TX}}^{\prime}\right)\left(1-\delta_{\left\{\mathbf{u}=\mathbf{u}^{\prime}\right\}}\right)\right), \Delta_{\mathrm{TX}}=\mathbf{z}_{\mathrm{TX}}-\mathbf{z}_{\mathrm{TX}}^{\prime}$, and $\Delta_{\mathrm{RX}}=\mathrm{z}_{\mathrm{RX}}-\mathrm{z}_{\mathrm{RX}}^{\prime}$, in which $\Sigma_{\mathrm{RX}}$ and $\Sigma_{\mathrm{RX}}^{\prime}$ represent the covariance of the location of the receiver of the first and second link, respectively, and $\mathbf{z}_{\mathrm{RX}}-\mathbf{z}_{\mathrm{RX}}^{\prime}$ denotes the distance between the means of the location distribution of the RX of first and second link. Similar definitions hold for the TX. The covariance function (7) explicitly includes the uncertainty of the position of both the TX and RX of each link, so that highly uncertain positions have reduced impact on the covariance.

Note that the definition of the covariance function now accounts for the Gaussian nature of the shadowing as well as

\footnotetext{
${ }^{2}$ The kernel (6) is a product of two kernels each using a subvector of the input vector. This results in a valid kernel giving a positive semi-definite covariance matrix [14, Ch. 6.2]
} 

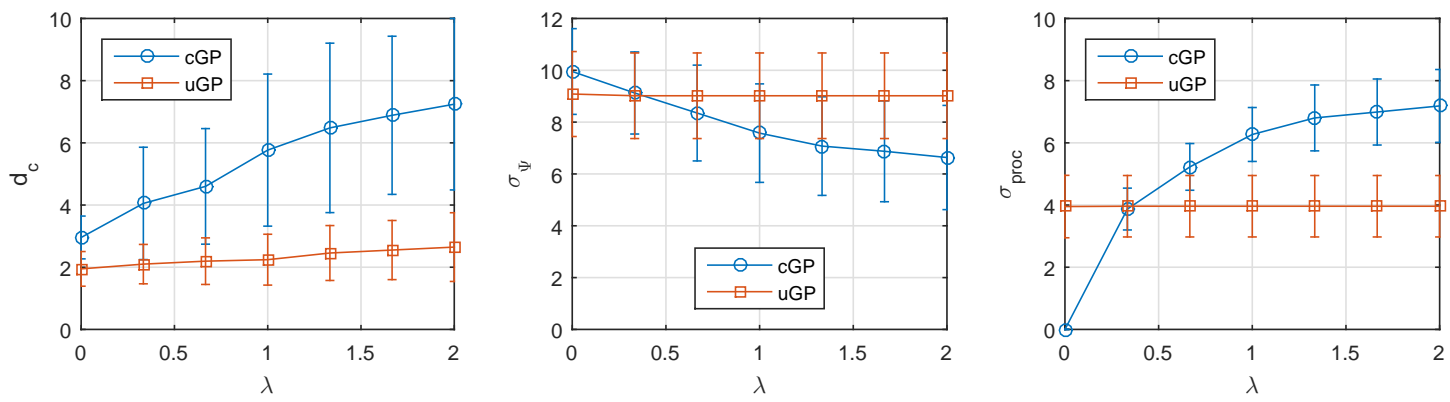

Fig. 2. Impact of average location error standard deviation $\lambda$ (in meters) on the model hyper-parameter estimation for cGP and uGP. The errorbars indicate one standard deviation. From left to right: estimated decorrelation distance $d_{c}$, estimated shadow standard deviation constant $\sigma_{\Psi}$, estimated process standard deviation modeling the kernel mismatch $\sigma_{\text {proc }}$.

the spatial correlation, but it does not capture the reciprocity of the channel.

\subsection{Introducing Channel Reciprocity in uGP}

With the definition of the input vector stated in uGP, an implicit ordering has been introduced, which has the effect that channel reciprocity is not ensured by the uGP framework itself. The incorporation of channel reciprocity into the uGP framework can be done: (i) by applying an operator to the input vector, (ii) by modifying the covariance function $[10, \mathrm{Ch}$. 4.2], (iii) by extending the database by its reciprocal counterpart. In this work, we have chosen the last approach, where for every measurement $y_{k}$ with associated $\mathbf{u}_{k}$, we add its reciprocal counterpart to the database (i.e., an additional entry with the same value $y_{k}$, but where the role of TX and RX in $\mathbf{u}_{k}$ are interchanged).

\subsection{Learning}

The uGP model hyper-parameter vector is given by $\theta=$ $\left[\sigma_{n}, \sigma_{\text {proc }}, d_{c}, L_{0}, \eta, \sigma_{\Psi}\right]^{\mathrm{T}}$. Learning refers to estimating the hyper-parameter vector $\theta$ from the training database $\{\mathbf{U}, \mathbf{y}\}$. Since $p(\mathbf{y} \mid \mathbf{U}, \theta)=\mathcal{N}_{\mathbf{y}}(\mu(\mathbf{U}), \mathbf{K})$, with $[\mathbf{K}]_{i j}=$ $k\left(\mathbf{u}_{i}, \mathbf{u}_{j}\right)+\sigma_{n}^{2} \delta_{\{i=j\}}$, a maximum likelihood estimator for $\theta$ is

$$
\hat{\theta}=\arg \max _{\theta} \log p(\mathbf{y} \mid \mathbf{U}, \theta) .
$$

In [15], it is stated that this likelihood function suffers from many local maxima and a Bayesian estimator is recommended instead. Nevertheless, in this work we estimate the hyper-parameters using (8) similar to [8].

\subsection{Prediction}

Assume we are in the possession of a training database $\{\mathbf{U}, \mathbf{y}\}$ and hyper-parameter vector $\theta$. For a test TX-RX location pair parametrized by $\mathbf{u}_{*}$, the predictive pdf
$p\left(P_{\mathrm{RX}}\left(\mathbf{u}_{*}\right) \mid \mathbf{y}, \mathbf{U}, \mathbf{u}_{*} ; \theta\right)$ follows a normal distribution with mean and variance given by [14, Ch. 6.4], [17]

$$
\begin{aligned}
\bar{P}_{\mathrm{RX}}\left(\mathbf{u}_{*}\right) & =\mu\left(\mathbf{u}_{*}\right)+\mathbf{k}_{*}^{\mathrm{T}} \mathbf{K}^{-1}(\mathbf{y}-\mu(\mathbf{U})), \\
\operatorname{Var}_{\mathrm{RX}}\left(\mathbf{u}_{*}\right) & =k_{* *}-\mathbf{k}_{*}^{\mathrm{T}} \mathbf{K}^{-1} \mathbf{k}_{*},
\end{aligned}
$$

where $\mathbf{k}_{*}=\left[k\left(\mathbf{u}_{1}, \mathbf{u}_{*}\right), k\left(\mathbf{u}_{2}, \mathbf{u}_{*}\right), \ldots, k\left(\mathbf{u}_{N}, \mathbf{u}_{*}\right)\right]^{\mathrm{T}}$ computed from (7), $\mu(\mathbf{U})=\left[\mu\left(\mathbf{u}_{1}\right), \mu\left(\mathbf{u}_{2}\right), \ldots, \mu\left(\mathbf{u}_{N}\right)\right]^{\mathrm{T}}$ computed from (3), and $k_{* *}=k\left(\mathbf{u}_{*}, \mathbf{u}_{*}\right)+\sigma_{n}^{2}$.

\section{NUMERICAL EXAMPLE}

We present performance results for learning the hyperparameters and its impact on the prediction performance for cGP and uGP under location uncertainty.

\subsection{Setup}

For convenience, we will ignore path-loss (so the field becomes zero-mean), and consider a field over a one-dimensional $(D=1)$ space of $15 \mathrm{~m}$ length: $P_{\mathrm{RX}}(\mathbf{x}):[0,15] \times[0,15] \rightarrow$ $\mathbb{R}$. The field is generated using a 2-dimensional GP with (6), setting $p=1, d_{c}=3, \sigma_{\Psi}=10$, and $\sigma_{n}=0.01$. Reciprocity is ensured by generating the field only for $\mathbf{q}_{\mathrm{TX}} \geq \mathbf{q}_{\mathrm{RX}}$ and then copying the values for $\mathbf{q}_{\mathrm{TX}}<\mathbf{q}_{\mathrm{RX}}$. $^{3}$ The field is sampled with a resolution of $37.5 \mathrm{~cm}$, leading to possible $40 \mathrm{TX}$ and RX locations, and thus a total of 1600 samples. The training set contains $N=250$ samples (including their reciprocal copies) randomly drawn from these 1600 samples and perturbed by location uncertainty. Similar to [8], we consider heterogeneous location errors with $\Sigma_{i}=\sigma_{i}^{2} \mathbf{I}$, where $\sigma_{i}$ is drawn from an exponential distribution with average location error standard deviation $\lambda$. The hyper-parameter vector is $\theta=\left[d_{c}, \sigma_{\Psi}, \sigma_{\text {proc }}\right]^{\mathrm{T}}$, where we assumed the agents know $\sigma_{n}$. The cGP method uses the covariance function (6) with

\footnotetext{
${ }^{3}$ Note that this procedure is not possible for $D>1$, as there is no absolute ordering of positions. In practice, this is irrelevant, since the measurements would be obtained from real data, which will inherently satisfy the reciprocity condition.
} 

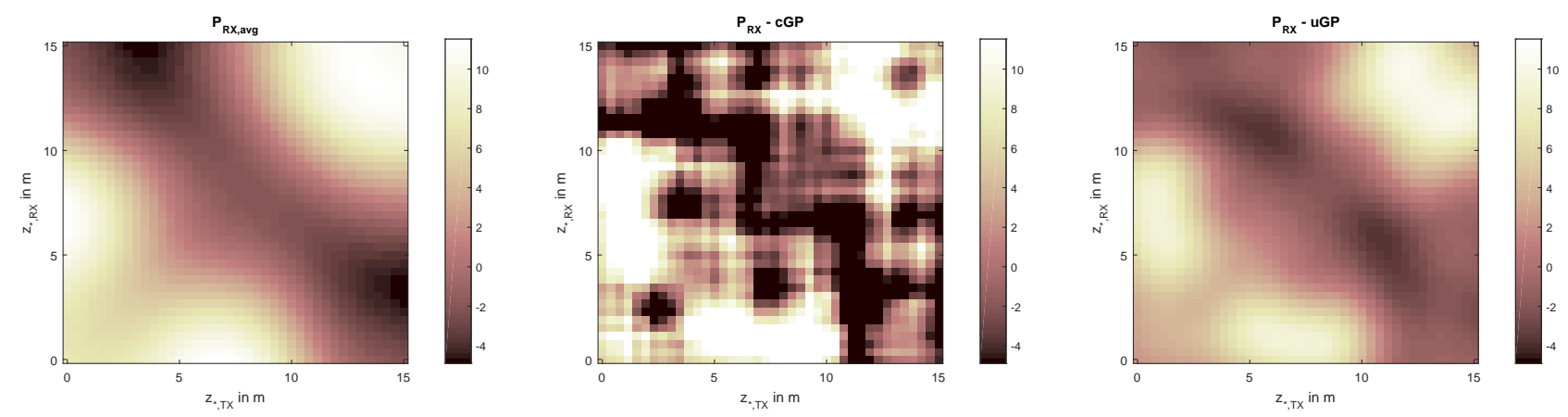

Fig. 3. Left: expected received power $P_{\mathrm{RX} \text {,avg }}$ (in $\mathrm{dBm}$ ) as a function of the expected position $\mathbf{z}_{*}$ for one realization of the spatially correlated shadowing field. Middle: reconstructed field using cGP method. Right: reconstructed field using uGP method. Note that in all panels, the fields are symmetric along the diagonal $\left(\mathbf{z}_{*, T X}=\mathbf{z}_{*, \mathrm{RX}}\right)$, i.e., the channel is reciprocal.

$p=1$ and uGP uses the expected covariance function (7). Simulation results are averaged over 30 realizations of the shadowing field.

\subsection{Results}

Learning: In Fig. 2, the impact of location uncertainty in the training set (parametrized by $\lambda$ ) on the model hyperparameter estimation for cGP and uGP is demonstrated. For cGP, we observe that the decorrelation distance $d_{c}$ increases as $\lambda$ increases. At the same time, $\sigma_{\text {proc }}$, which captures input uncertainty, increases as well. As a consequence, $\sigma_{\Psi}$ decreases as the signal variance needs to be maintained. It is given by the relation $\sigma_{\Psi}^{2}=\sigma_{\text {tot }}^{2}-\sigma_{n}^{2}-\sigma_{\text {proc }}^{2}$, where the variance of the received signal power is estimated from measurements $\mathbf{y}$ of the training set by $\sigma_{\text {tot }}^{2}=\|\mathbf{y}\|^{2} / N$. This behavior can be observed in the middle panel of Fig. 2. In the case of uGP, the estimated hyper-parameter $d_{c}$ stays almost constant among different levels of location uncertainty in the training set. The uGP method exploits the fact that location uncertainty of the $i$-th training sample $\mathbf{x}_{i}$ is provided by its distribution parameters $\mathbf{u}_{i}$. Hence, there is no need to increase $d_{c}$ with increasing $\lambda$ since the kernel function (7) directly works on $\mathbf{u}_{i}$ in contrast to $\mathbf{x}_{i}$ as is the case for cGP. The hyper-parameter $\sigma_{\text {proc }}$ captures the kernel mismatch between kernel (6) used to generate the true field and the uGP-kernel function (7). This mismatch is constant and independent of $\lambda$ and is reflected by the estimated values of $\sigma_{\text {proc }}$ shown in the right panel of Fig. 2. Furthermore, for a present location uncertainty $\lambda$, a constant $\sigma_{\text {proc }}$ implies also a constant $\sigma_{\Psi}$. This can be observed in the middle panel of the same figure.

Prediction: In Fig 3, we visualize the prediction performance for the case that only the test set has uncertain location information (with $\sigma=2$ ), while training data had $\lambda=0$. The learned hyper-parameters can be extracted from Fig. 2. Similar to [8], the performance must be assessed with respect to the expected received power $P_{\mathrm{RX}, \mathrm{avg}}\left(\mathbf{u}_{*}\right)=$ $\int P_{\mathrm{RX}}\left(\mathbf{x}_{*}\right) p\left(\mathbf{x}_{*}\right) \mathrm{d} \mathbf{x}_{*}$, where $p\left(\mathbf{x}_{*}\right)=\mathcal{N}_{\mathbf{x}_{*}}\left(\mathbf{z}_{*}, \sigma^{2} \mathbf{I}\right)$ and the mean value of $\mathbf{x}_{*}$ can be written $\mathbf{z}_{*}=\left[\mathbf{z}_{*, \mathrm{TX}}, \mathbf{z}_{*, \mathrm{RX}}\right]^{\mathrm{T}}$. In the left panel of Fig. 3, we plot one realization of the average true field $P_{\mathrm{RX} \text {,avg }}$ for different mean locations of the transmitter $\mathbf{z}_{*, \mathrm{TX}}$ and receiver $\mathbf{z}_{*, \mathrm{RX}}$. Note the symmetry of the field along its diagonal, due to channel reciprocity. The middle and right panel in Fig. 3 illustrate the predicted mean $\bar{P}_{\mathrm{RX}}$ using cGP and uGP, respectively. We observe that due to our design, the channel reciprocity property holds. We clearly see that uGP provides a much better estimate of the true expected field than cGP. The predicted mean $\bar{P}_{\mathrm{RX}}$ using cGP varies significantly with small variations of the TX and RX location. In contrary to this, the predicted mean using uGP is a smooth function over the TX and RX location. Due to space limitations, we did not include results where both training and test data were subject to uncertainty. However we expect similar gains as in [8]. Finally, we expect that when the mean function is included, the gains of uGP over cGP will be even more pronounced.

\section{CONCLUSIONS}

We presented a framework to incorporate transmitter and receiver location uncertainty for channel gain prediction of a spatially correlated shadowing field, which can be utilized by, e.g., cooperating agents to enable end-to-end communication connectivity. The proposed framework is based on Gaussian processes, where the input variable is the location distribution of the agents, instead of the locations. This allows us to explicitly account for varying levels of location uncertainty during both training and testing. Simulation results indicate improved performance when learning hyper-parameters and predicting the received powers, as compared to methods that neglect location uncertainty. For future work, we plan to support our findings by experiments using different types of radio communication and positioning technologies and by including this channel prediction framework in control applications. 


\section{REFERENCES}

[1] Jeff S. Shamma, Ed., Cooperative control of distributed multi-agent systems, Wiley Online Library, 2007.

[2] Tugay Eyceoz, Alexandra Duel-Hallen, and Hans Hallen, "Deterministic channel modeling and long range prediction of fast fading mobile radio channels," IEEE Communications Letters, vol. 2, no. 9, pp. 254256, 1998.

[3] Andrea Goldsmith, Wireless Communications, Cambridge University Press, 2005.

[4] Annika Böttcher, Peter Vary, Christian Schneider, and Reiner S. Thomä, "De-correlation distance of the large scale parameters in an urban macro cell scenario," in 6th European Conference on Antennas and Propagation (EUCAP), 2012, pp. 1417-1421.

[5] Niklas Jalden, Analysis and Modelling of Joint Channel Properties from Multi-site, Multi-Antenna Radio Measurements, Ph.D. thesis, KTH, Signal Processing, 2010.

[6] Mikael Gudmundson, "Correlation model for shadow fading in mobile radio systems," Electronics Letters, vol. 27, no. 23, pp. 2145-2146, 1991.

[7] Zhenyu Wang, Eustace K. Tameh, and Andrew R. Nix, "Joint Shadowing Process in Urban Peer-to-Peer Radio Channels," Vehicular Technology, IEEE Transactions on, vol. 57, no. 1, pp. 52-64, 2008.

[8] L. Srikar Muppirisetty, Tommy Svensson, and Henk Wymeersch, "Spatial Wireless Channel Prediction under Location Uncertainty," to appear in IEEE Transactions on Wireless Communications, 2015.

[9] Piyush Agrawal and Neal Patwari, "Correlated link shadow fading in multi-hop wireless networks," IEEE Transactions on Wireless Communications, vol. 8, no. 8, pp. 4024-4036, 2009.

[10] Jonathan Fink, Communication for teams of networked robots, Ph.D. thesis, Penn, University of Pennsylvania, 2011.

[11] Mehrzad Malmirchegini and Yasamin Mostofi, "On the spatial predictability of communication channels," IEEE Transactions on Wireless Communications, vol. 11, no. 3, pp. 964-978, 2012.

[12] Yuan Yan and Yasamin Mostofi, "Impact of localization errors on wireless channel prediction in mobile robotic networks," in IEEE Globecom, Workshop on Wireless Networking for Unmanned Autonomous Vehicles, 2013, pp. 1374-1379.
[13] S.S. Szyszkowicz, H. Yanikomeroglu, and J.S. Thompson, "On the feasibility of wireless shadowing correlation models," Vehicular Technology, IEEE Transactions on, vol. 59, no. 9, pp. 4222-4236, 2010.

[14] Christopher M. Bishop, Pattern Recognition and Machine Learning, Springer, 2006.

[15] Patrick Dallaire, Camille Besse, and Brahim Chaibdraa, "An approximate inference with Gaussian process to latent functions from uncertain data," Neurocomputing, vol. 74, no. 11, pp. 1945-1955, 2011.

[16] Agathe Girard, Approximate methods for propagation of uncertainty with Gaussian process models, Ph.D. thesis, University of Glasgow, 2004.

[17] Carl Edward Rasmussen and Christopher K. I. Williams, Gaussian processes for machine learning, MIT Press, 2006. 